\title{
Performance of Cooperative Spectrum Sensing over Non-Identical Fading Environments
}

\author{
Anlei Rao, Student Member, IEEE and Mohamed-Slim Alouini, Fellow, IEEE
}

\begin{abstract}
Different from previous works in cooperative spectrum sensing that assumed the sensing channels independent identically distributed (i.i.d.), we investigate in this paper the independent but not identically distributed (i.n.i.d.) situations. In particular, we derive the false-alarm probability and the detection probability of cooperative spectrum sensing with the scheme of energy fusion over i.n.i.d. Rayleigh, Nakagami, and Rician fading channels. From the selected numerical results, we can see that cooperative spectrum sensing still gives considerably better performance even over i.n.i.d. fading environments.
\end{abstract}

Index Terms-Cooperative spectrum sensing; Energy detection; Nakagami fading; Rician fading;

\section{INTRODUCTION}

The dramatic growth of wireless services over the last decade explains the great demand for radio spectrum. The spectrum resource, however, is limited and most of it has already been allocated. On the other hand, according to the report of the Federal Communications Commission (FFC) [1], many portions of the allocated spectrum are not fully utilized. In order to deal with this conflict between spectrum scarcity and spectrum under-utilization, cognitive radio has been proposed as a revolutionary technology for the next generation of wireless communication networks [2].

To guarantee that the operation of the primary users is not affected, the secondary users must possess the ability of sensing the presence of active primary users, and this process is known as spectrum sensing. In some specific environment, multi-path fading and shadowing may cause the disability of secondary users to detect a primary user. In order to solve such problems, multiple secondary users can cooperate with each other to conduct the spectrum sensing to achieve an improved performance [3].

Recently, cooperative sensing has been widely studied [4][5], and these studies suppose that the sensing channels are independent identically distributed (i.i.d.). But this is not always the case in practice. Indeed in many instances, the received signals by cooperating secondary users may experience a variety of path loss, shadowing or fading conditions. As such, a more appropriate and practical assumption is to consider independent but not identically distributed (i.n.i.d.) channels. In this paper, we consider cooperative sensing in such kind of environment and we assume that the reporting

This work was supported by King Abdullah University of Science and Technology (KAUST). This is an extended version of the work which was accepted for presentation at IEEE Vehicular Technology Conference (VTC'11), Budapest, Hungary, May, 2011.

Anlei Rao and M.-S. Alouini are with the Division of Physical Sciences and Engineering, KAUST, 23955-6900, KSA. Email: \{anlei.rao, slim.alouini\}@kaust.edu.sa channels to the fusion center are error-free. In particular, instead of sending the binary local decision, each secondary user is assumed to transmit the normalized energy statistic to the fusion center, which makes a final decision by fusing these local energy statistics. In this context, we calculate the false-alarm probability and miss-detection probability over i.n.i.d. Rayleigh, Nakagami, and Rician fading channels with a threshold determined by the Neyman-Pearson criterion.

The rest of this paper is organized as follows. In Section II, the system model is introduced. Next, Section III presents the performance analysis over i.n.i.d. Rayleigh, Nakagami, and Rician fading channels. Then the following section shows some selected numerical results. Finally, conclusions is presented in Section V.

\section{SySTEM MODEL}

We consider a cooperative cognitive radio network with $N$ secondary users and a fusion center, and assume that each secondary user performs local energy sensing independently and report these energy values to the fusion center over errorfree reporting channels. These cooperating secondary users are uniformly distributed in a cell of radius $R$ centered around the primary user, and in this case, the average SNR $\bar{\gamma}$ for each sensing channel is assumed to be log-normal distributed with a shadow standard deviation of $\theta \mathrm{dB}$, an average SNR $\bar{\gamma}_{R}$ at distance $R$, and a mean value following an exponentially decreasing path loss model with an exponent $\alpha$. In this case, the probability density function (PDF) of average signal-tonoise ratio (SNR) $\bar{\gamma}$ is given by [6], [7]:

$f_{\bar{\gamma}}(\bar{\gamma})=\frac{2}{c} \exp \left\{\frac{2 \theta^{2}-2 c\left(\bar{\gamma}-\bar{\gamma}_{R}\right)}{c^{2}}\right\} Q\left(\frac{2 \theta^{2}-c\left(\bar{\gamma}-\bar{\gamma}_{R}\right)}{c \cdot \theta}\right)$,

where $c=10 \alpha \log (e)$ is the exponential path loss parameter, $Q(\cdot)$ is the $Q$-function defined as the tail probability of the standard normal distribution, i.e. $Q(x)=$ $\frac{1}{\sqrt{2 \pi}} \int_{x}^{\infty} \exp \left(-\frac{u^{2}}{2}\right) d u$.

The sensing channels for the secondary users are assumed to be independent to each other. Different from previous works, however, these channels may be not identically distributed but subject to the same kind of fading. With such an i.n.i.d. assumption, the SNR of the received signal from the primary user for $n$-th secondary user can be denoted as $\gamma_{n}$, with its mean $\bar{\gamma}_{n}$, the distribution of which is subject to (1), and PDF $f_{\gamma_{n}}\left(\gamma_{n}\right)$. The $n$-th secondary user measures the normalized energy statistic of the signal it has received as $E_{n}$, which has a central chi-square distribution under the hypothesis $H_{0}$ (inactive primary user) and non-central chi-square distribution with a center parameter $2 \gamma_{n}$ under the hypothesis $H_{1}$ (active 
primary user) [8]:

$$
E_{n} \sim \begin{cases}\chi_{2 u}^{2} & H_{0} \\ \chi_{2 u}^{2}\left(2 \gamma_{n}\right) & H_{1},\end{cases}
$$

where $u=T W$ is the time bandwidth product with $T$ the observation time interval and $W$ the one-sided bandwidth.

The fusion center collects the normalized energy statistic from each secondary user using the energy fusion scheme [4] to make a decision. Under perfect reporting channel conditions, the fusion energy statistic yields

$$
E=\sum_{n=1}^{N} E_{n}
$$

Based on (3), it is not difficult to show that the fusion energy statistic $E$ is also subject to a central chi-square distribution under the hypothesis $H_{0}$ and a non-central chisquare distribution with a center parameter $2 \gamma$ under the hypothesis $H_{1}$ :

$$
E \sim \begin{cases}\chi_{2 N u}^{2} & H_{0} \\ \chi_{2 N u}^{2}(2 \gamma) & H_{1}\end{cases}
$$

where $\gamma=\sum_{n=1}^{N} \gamma_{n}$ is the combined SNR with a PDF $f_{\gamma}(\gamma)$. As such, the PDF of $E$ conditioned on $\gamma$ is given by

$$
f_{E}(x)= \begin{cases}\frac{1}{2^{N u} \Gamma(N u)} x^{N u-1} \mathrm{e}^{-\frac{x}{2}} & H_{0} \\ \frac{1}{2}\left(\frac{x}{2 \gamma}\right)^{\frac{N u-1}{2}} \mathrm{e}^{-\frac{2 \gamma+x}{2}} I_{N u-1}(\sqrt{2 \gamma x}) & H_{1} \quad,\end{cases}
$$

where $\Gamma(\cdot)$ is the gamma function and $I_{M}(\cdot)$ is the $M$-th order modified Bessel function of the first kind.

To make a decision, the fusion center compares the energy statistic $E$ to a threshold $\lambda$, and decide on $H_{0}$ when $E<\lambda$ and on $H_{1}$ when $E>\lambda$. In this conditions, the false-alarm and detection probability conditioned on $\gamma$ are given by [8]

$$
\begin{aligned}
& P_{f}=\frac{\Gamma(N u, \lambda / 2)}{\Gamma(N u)}, \\
& P_{d}=Q_{N u}(\sqrt{2 \gamma}, \sqrt{\lambda}),
\end{aligned}
$$

where $\Gamma(\cdot, \cdot)$ is the upper incomplete gamma function, and $Q_{M}(a, b)$ is the generalized Marcum Q-function, i.e. $Q_{M}(a, b)=\frac{1}{a^{M-1}} \int_{b}^{\infty} x^{M} \exp \left(-\frac{x^{2}+a^{2}}{2}\right) I_{M-1}(a x) d x$. The averaged detection probability can then be computed in different fading environments as

$$
\bar{P}_{d}=\int_{0}^{+\infty} Q_{N u}(\sqrt{2 \gamma}, \sqrt{\lambda}) f_{\gamma}(\gamma) d \gamma
$$

since the conditional false-alarm probability is independent of $\gamma$, then $\bar{P}_{f}=P_{f}$.

The threshold $\lambda$ can be determined using the NeymanPearson criterion. In particular, given a desired false-alarm probability $P_{f}=\alpha$, the threshold $\lambda$ can be determined and can be used in (6) to find the average detection probability.

\section{Performance AnAlysis}

\section{A. Nakagami Fading Channels}

Nakagami fading occurs for multipath scattering with relatively large delay-time spreads and with different clusters of reflected waves. It represents a wide range of multipath channels via the fading parameter $m$ [9]. For instance, the Nakagami fading includes the one-sided Gaussian fading ( $m=1 / 2$, which corresponds to worst-case fading) and the Rayleigh fading $(m=1)$ as special cases.

For Nakagami fading channels, the local SNR of secondary users is subject to a Gamma distribution given by

$f\left(\gamma_{n}\right)=\frac{1}{\Gamma\left(m_{n}\right)}\left(\frac{m_{n}}{\bar{\gamma}_{n}}\right)^{m_{n}} \gamma_{n}{ }^{\left(m_{n}-1\right)} \exp \left\{-\frac{m_{n}}{\bar{\gamma}_{n}} \gamma_{n}\right\}, \gamma_{n}>0$,

where $m_{n}$ is the Nakagami parameter for the $n$-th sensing channel.

For distinct $\bar{\gamma}_{n}$ 's, the PDF of the combined SNR $\gamma=$ $\sum_{n=1}^{N} \gamma_{n}$ in the case is given by

$f(\gamma)=\sum_{n=1}^{N} \sum_{j=1}^{m_{n}} \frac{A_{n j}}{\Gamma(j)}\left(\frac{m_{n}}{\bar{\gamma}_{n}}\right)^{m_{n}} \gamma^{j-1} \exp \left\{-\frac{m_{n}}{\bar{\gamma}_{n}} \gamma\right\}, \gamma>0$,

where $A_{n j}$ is determined by

$A_{n j}=\left.\frac{(-1)^{m_{n}-j}}{\left(m_{n}-j\right) !} \frac{d^{\left(m_{n}-j\right)}}{d s^{\left(m_{n}-j\right)}}\left\{M_{\gamma}(s)\left(1-\frac{\bar{\gamma}_{n}}{m_{n}} s\right)^{m_{n}}\right\}\right|_{s=\frac{m_{n}}{\bar{\gamma}_{n}}}$

with $M_{\gamma}(\cdot)$ given in the case by

$$
M_{\gamma}(s)=\left\{\prod_{n=1}^{N}\left(1-\frac{\bar{\gamma}_{n}}{m_{n}} s\right)^{-m_{n}}\right\} .
$$

Inserting (7) in (6), we get the average detection probability with the help of Eq.(29) in [10] as

$$
\bar{P}_{d \_N a k}=\sum_{n=1}^{N} \sum_{j=1}^{m_{n}} \frac{A_{n j}}{2^{j-1} \Gamma(j)}\left(\frac{m_{n}}{\bar{\gamma}_{n}}\right)^{m_{n}} G_{N u},
$$

where $G_{N u}$ can be determined recursively as

$$
\begin{aligned}
G_{M}= & G_{M-1}+\frac{\Gamma(j) \lambda^{M-1} e^{-\frac{\lambda}{2}}}{2^{M-j}(M-1) !}\left(1+\frac{m_{i}}{\bar{\gamma}_{n}}\right)^{-j} \\
& \times{ }_{1} F_{1}\left(j ; M ; \frac{\lambda}{2} \frac{\bar{\gamma}_{n}}{\bar{\gamma}_{n}+m_{n}}\right),
\end{aligned}
$$

with the initial $G_{1}$ given by

$$
G_{1}=\int_{0}^{\infty} x^{2 j-1} \exp \left\{-\frac{m_{n}}{2 \bar{\gamma}_{n}} x^{2}\right\} Q(x, \sqrt{\lambda}) d x,
$$

and ${ }_{1} F_{1}(\cdot ; \cdot ; \cdot)$ is the confluent hypergeometric function. $G_{1}$ can be written in a closed form with the help of $[10,25]$ :

$$
\begin{aligned}
G_{1}= & \frac{2^{j-1} \Gamma(j) \bar{\gamma}_{n}^{j+1}}{\left(m_{n}+\bar{\gamma}_{n}\right) m_{n}^{j}} \exp \left\{-\frac{\lambda}{2} \frac{m_{n}}{m_{n}+\bar{\gamma}_{n}}\right\} \times \\
& {\left[\sum_{k=0}^{j-2}\left(\frac{m_{n}}{m_{n}+\bar{\gamma}_{n}}\right)^{k} L_{k}\left(-\frac{\lambda}{2} \frac{\bar{\gamma}_{n}}{m_{n}+\bar{\gamma}_{n}}\right)+\right.} \\
& \left.\left(1+\frac{m_{n}}{\bar{\gamma}_{n}}\right)\left(\frac{m_{n}}{m_{n}+\bar{\gamma}_{n}}\right)^{j-1} L_{j-1}\left(-\frac{\lambda}{2} \frac{\bar{\gamma}_{n}}{m_{n}+\bar{\gamma}_{n}}\right)\right],
\end{aligned}
$$


where $L_{k}(\cdot)$ is the Laguerre polynomials with degree $k$.

As a special case, the average detection probability for Rayleigh fading channels is given by making all $m_{n}=1$ in (8) resulting in

$$
\begin{aligned}
\bar{P}_{d_{-} \text {Ray }} & =\sum_{n=1}^{N} A_{n}\left\{\frac{\Gamma\left(N u-1, \frac{\lambda}{2}\right)}{\Gamma(N u-1)}+\exp \left(-\frac{\lambda}{2\left(1+\bar{\gamma}_{n}\right)}\right)\right. \\
& \left.\times P\left(N u-1, \frac{\lambda}{2} \frac{\bar{\gamma}_{n}}{\left(1+\bar{\gamma}_{n}\right)}\right)\left(1+\frac{1}{\bar{\gamma}_{n}}\right)^{N u-1}\right\},
\end{aligned}
$$

where $P(M, x)=\gamma(M, x) / \Gamma(M)$ with $\gamma(M, x)=\Gamma(M)-$ $\Gamma(M, x)$ as the lower incomplete gamma function, and $A_{n}$ is determined by

$$
A_{n}=\left.M_{\gamma}(s)\left(1-\bar{\gamma}_{n} s\right)\right|_{s=1 / \bar{\gamma}_{n}},
$$

where $M_{\gamma}(s)=\left\{\prod_{n=1}^{N}\left(1-\bar{\gamma}_{n} s\right)\right\}^{-1}$.

\section{B. Rician Fading Channels}

Rician fading occurs when one of the paths, typically a line of sight signal, is much stronger than the others. It is described by two parameters: $k$, which is the ratio between the power in the direct path and the power in other scattered paths, and $\bar{\gamma}$, the power in the direct path, which acts as a scaling factor to its distribution. When there is no line of sight signal, the Rician fading reduces to Rayleigh fading as its special case $(k=0)$.

For Rician fading channels, each secondary user's local SNR is subject to a weighted non-central chi-square distribution with two degree of freedom given by

$$
\begin{aligned}
f\left(\gamma_{n}\right) & =\frac{k_{n}+1}{\bar{\gamma}_{n}} \exp \left\{-k_{n}-\frac{\left(k_{n}+1\right) \gamma_{n}}{\bar{\gamma}_{n}}\right\} \\
& \times I_{0}\left(2 \sqrt{\frac{k_{n}\left(k_{n}+1\right) \gamma_{n}}{\bar{\gamma}_{n}}}\right), \quad \gamma_{n}>0,
\end{aligned}
$$

where $k_{n}$ is the Rician factor for the $n$-th channel. With distinct $\bar{\gamma}_{n}$, the PDF of the combined SNR $\gamma=\sum_{n=1}^{N} \gamma_{n}$ is given by [11] as

$$
\begin{aligned}
f_{\gamma}(\gamma) & =\frac{\gamma^{N-1}}{\Gamma(N)(2 \beta)^{N}} \exp \left\{-\frac{\gamma}{2 \beta}\right\} \\
& \times \sum_{n \geq 0} \frac{n ! \cdot c_{n}}{(N)_{n}} L_{n}^{(N-1)}\left(\frac{N \gamma}{2 \beta \mu_{0}}\right), \quad \forall \mu_{0}>0,
\end{aligned}
$$

where $L_{n}^{(\alpha)}(x)$ is the generalized Laguerre polynomials given by $L_{n}^{(\alpha)}(x)=\sum_{i=1}^{n}\left(\begin{array}{c}n+\alpha \\ n-i\end{array}\right)(-1)^{i} x^{i} / i !,(N)_{n}=N \cdot(N-$ 1) $\cdots(N-n+1), \lambda_{n}=2 k_{n}, \beta_{n}=\frac{\bar{\gamma}_{n}}{2+\lambda_{n}}, \beta=\sum_{n=1}^{N} \beta_{n}$, and $c_{n}=\left(-\mu_{0}\right)^{n} g^{(n)}\left(\mu_{0}\right) / n$ ! with

$g(\mu)=\exp \left\{-\frac{1}{2} \sum_{n=1}^{N} \frac{\lambda_{n} \beta_{n}(N-\mu)}{\beta \mu+\beta_{n}(N-\mu)}\right\} \prod_{n=1}^{N} \frac{N \beta}{\beta \mu+\beta_{n}(N-\mu)}$

In (10), the coefficients $c_{n}$ are computed in a recursive fashion:

$$
c_{n}=\frac{1}{n} \sum_{j=0}^{n-1} c_{j} \cdot d_{n-j}, \quad n>0
$$

$$
\begin{gathered}
c_{0}=\left(\frac{N}{\mu_{0}}\right)^{N} \exp \left\{-\frac{1}{2} \sum_{n=1}^{N} \frac{\lambda_{n} \beta_{n}\left(N-\mu_{0}\right)}{\beta \mu_{0}+\beta_{n}\left(N-\mu_{0}\right)}\right\} \\
\times \prod_{n=1}^{n}\left(1+\frac{\beta_{n}}{\beta}\left(\frac{N}{\mu_{0}}-1\right)\right)^{-1}, \\
d_{j}=-\frac{j \beta N}{2 \mu_{0}} \sum_{n=1}^{N} \frac{\lambda_{n} \beta_{n}}{\left(\beta-\beta_{n}\right)^{2}}\left(\frac{\mu_{0}\left(\beta-\beta_{n}\right)}{\beta \mu_{0}+\beta_{n}\left(N-\mu_{0}\right)}\right)^{j+1} \\
+\sum_{n=1}^{N}\left(\frac{1-\beta_{n} / \beta}{1+\left(\beta_{n} / \beta\right)\left(N / \mu_{0}-1\right)}\right)^{j}, j>0 .
\end{gathered}
$$

Inserting (10) in (6) and using [10, 29], we get the average detection probability as

$$
\begin{aligned}
\bar{P}_{d_{-} R i c}= & \frac{1}{\Gamma(N)(2 \beta)^{N}} \sum_{n \geq 0} \frac{n ! \cdot c_{n}}{(N)_{n}} \times \\
& \sum_{i=0}^{n}(-1)^{i} \frac{1}{2^{N+i-1}} \frac{\mu^{i}}{i !}\left(\begin{array}{c}
N+n-1 \\
n-i
\end{array}\right) G_{N u},
\end{aligned}
$$

where $\mu=\frac{N}{2 \beta \mu_{0}}$ with $\mu_{0} \geq N$, and $G_{N u}$ can be determined recursively using

$$
\begin{aligned}
G_{M}=G_{M-1}+ & \frac{\Gamma(N+i) \lambda^{M-1} e^{-\frac{\lambda}{2}}}{2^{M-N-i} \Gamma(M)(p+1)^{N+i}} \\
& \times{ }_{1} F_{1}\left(N+i ; M ; \frac{\lambda}{2(p+1)}\right)
\end{aligned}
$$

with $p=\frac{1}{2 \beta}$, and the initial $G_{1}$ given by

$$
G_{1}=\int_{0}^{\infty} x^{2(N+i)-1} \exp \left\{-\frac{p}{2} x^{2}\right\} Q(x, \sqrt{\lambda}) d x
$$

which can be computed in closed-form with the help of [10, 25] as

$$
\begin{aligned}
G_{1}= & \frac{2^{N+i-1} \Gamma(N+i)}{(p+1) p^{N+i}} \exp \left\{-\frac{\lambda}{2} \frac{p}{p+1}\right\} \\
& \times\left[\sum_{k=0}^{N+i-2}\left(\frac{p}{p+1}\right)^{k} L_{k}\left(-\frac{\lambda}{2} \frac{1}{p+1}\right)\right. \\
& \left.+\frac{p^{N+i-1}}{(1+p)^{N+i-2}} L_{N+i-1}\left(-\frac{\lambda}{2} \frac{1}{p+1}\right)\right] .
\end{aligned}
$$

\section{NUMERICAL RESULTS}

Performance of the network is often described through its receive operating characteristic (ROC) curves $\left(P_{d}\right.$ versus $\left.P_{f}\right)$ or complementary ROC curves $\left(P_{m}=1-P_{d}\right.$ versus $\left.P_{f}\right)$ for different situations of interests. In the following figures, we sample the set of average $\operatorname{SNR}\left\{\bar{\gamma}_{n}, n=1,2, \cdots, N\right\}$ from (1) ${ }^{1}$, with the standard deviation $\theta=3 d B$ and the exponent of the path loss model $\alpha=3$, and plot the performance curves by a Monte Carlo method using the Neyman-Pearson criterion to calculate the threshold for a decision.

\footnotetext{
${ }^{1}$ In this paper, we generate the average $\operatorname{SNR}\left\{\bar{\gamma}_{i}\right\}$ 's as per (1) with the assumption that the users are uniformly distributed in the cell, and that the $\left\{\bar{\gamma}_{i}\right\}$ 's are independent. In reality, the $\left\{\bar{\gamma}_{i}\right\}$ 's may be dependent because of correlated shadowing. In this case, the $\left\{\bar{\gamma}_{i}\right\}$ 's should be sampled from

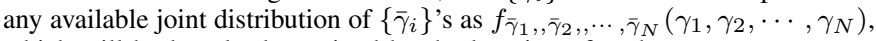
which will be largely determined by the location of each user.
} 


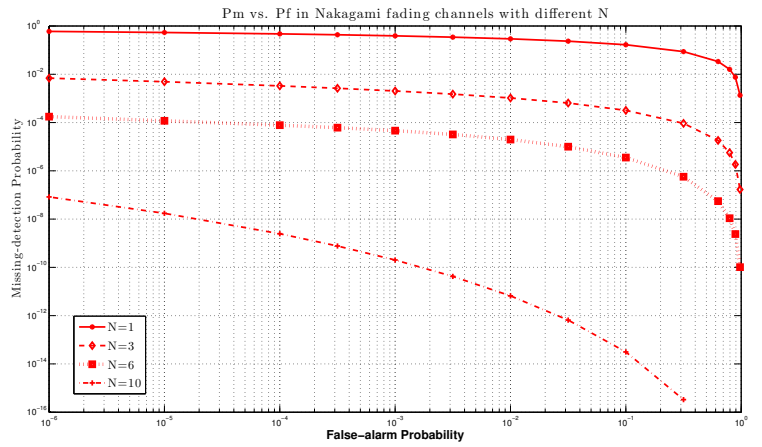

Fig. 1: Complementary ROC curves for Nakagami fading channels for different values of $N$ with a Nakagami parameter $m=3\left(\bar{\gamma}_{R}=8 \mathrm{~dB}\right.$ and $\left.u=5\right)$.

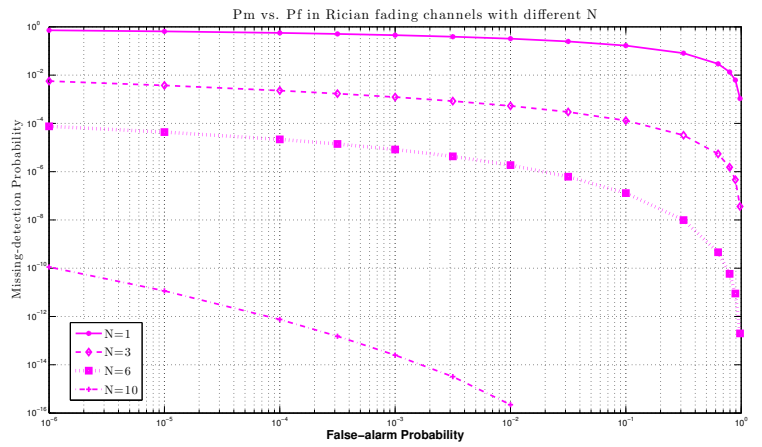

Fig. 2: Complementary ROC curves for Rician fading channel for different values of $N$ with a Rician factor $k=3$ $\left(\bar{\gamma}_{R}=8 \mathrm{~dB}\right.$ and $\left.u=5\right)$.

Ignoring the fading and noise of the reporting channels between the secondary users and the fusion center, we examine the performance of energy fusion over i.n.i.d. sensing channels with Nakagami and Rician fading, respectively. In Fig.1 and Fig.2, we generate the complementary ROC curves for Nakagami fading and Rician fading for different numbers $N$ of cooperative secondary users. Clearly the greater is the number of cooperative secondary users, the higher performance can the network achieve. From the figures, we can see that the miss-detection probability decreases greatly with the number of secondary users $N$ increasing slightly, which shows that cooperating improves the performance of sensing.

In Fig. 3 and Fig.4, we generate complementary ROC curves for Nakagami and Rician fading conditions for different average SNR $\bar{\gamma}_{R}$ at distance $R$. From the figures, we notice that there is a great improvement in the performance with several $\mathrm{dB}$ 's increment in $\bar{\gamma}_{R}$. The cooperating secondary users are distributed in a cell centered around the primary user. A higher value of $\bar{\gamma}_{R}$ means better conditions of the sensing channels between the secondary users and primary user, or means that these secondary users are distributed in a closer proximity to the primary user.

In Fig.5, we plot the complementary ROC curves for different values of Nakagami parameters over Nakagami fading channels. As we can see in the figure, there is an improvement

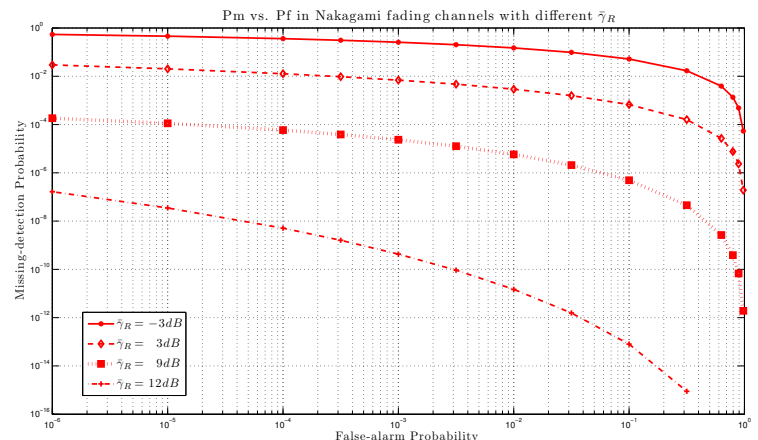

Fig. 3: Complementary ROC curves for Nakagami fading channel for different values of $\bar{\gamma}_{R}$ with a Nakagami parameter $m=3(N=5 \mathrm{~dB}$ and $u=5)$.

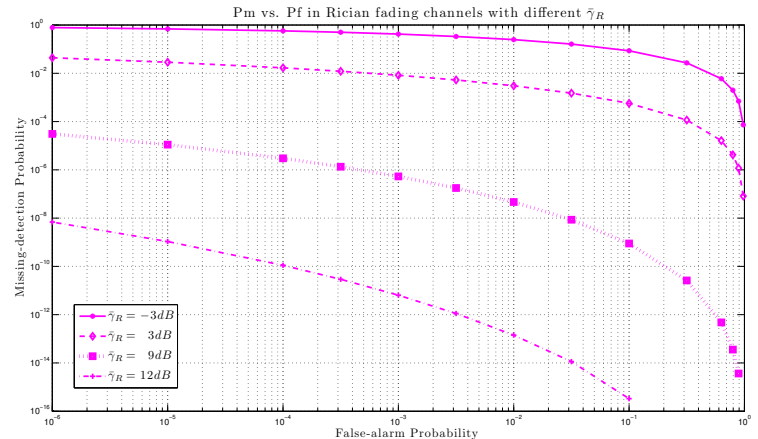

Fig. 4: Complementary ROC curves for Rician fading channel for different values of $N$ with a Rician factor $k=3$ $(N=5 \mathrm{~dB}$ and $u=5)$.

of roughly one order of magnitude for the miss-detection probability from $m=1$ to $m=2$. This improvement diminishes when $m$ is reduced by half and increases when $m$ doubled.

In Fig.6, we plot the complementary ROC curves for different values of Rician factors over Rician fading channels. The Rician factor $k$ is the ratio between the power in the direct path and the power in other scattered paths. Clearly the larger the Rician factor $k$ is, the better performance we can achieve. As we can find in this figure, the performance improves as the Rician factor $k$ increases.

\section{CONCLUSIONS}

In this paper, we analyzed the performance of cooperative spectrum sensing over the i.n.i.d. environments with Rayleigh, Nakagami and Rician fading. With the scheme of energy fusion, closed form of false-alarm probability and detection probability were derived. It was shown by selected numerical results that cooperative spectrum sensing still works considerably well in an i.n.i.d. environment.

\section{REFERENCES}

[1] P. Kolodzy, I. Avoidance, and S. Models, "Spectrum Policy Task Force," Federal Commun. Comm., Washington, DC, Rep. ET Docket, 2002. 


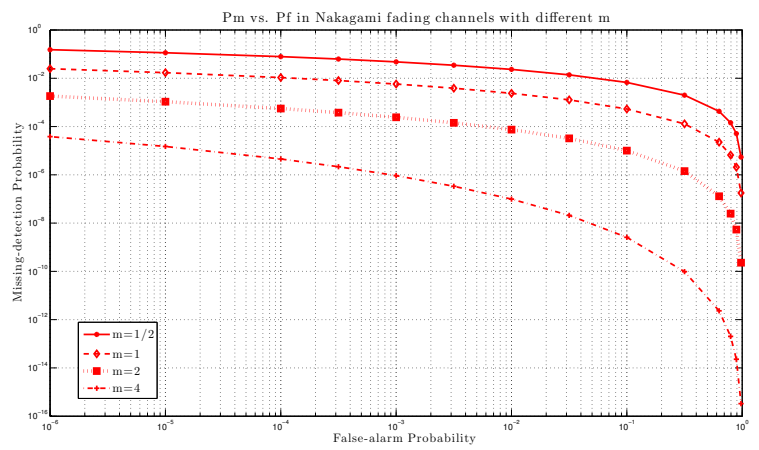

Fig. 5: Complementary ROC curves for Nakagami fading channel for different values of Nakagami parameter $m$ $\left(N=5, \bar{\gamma}_{R}=8 \mathrm{~dB}\right.$ and $\left.u=5\right)$.

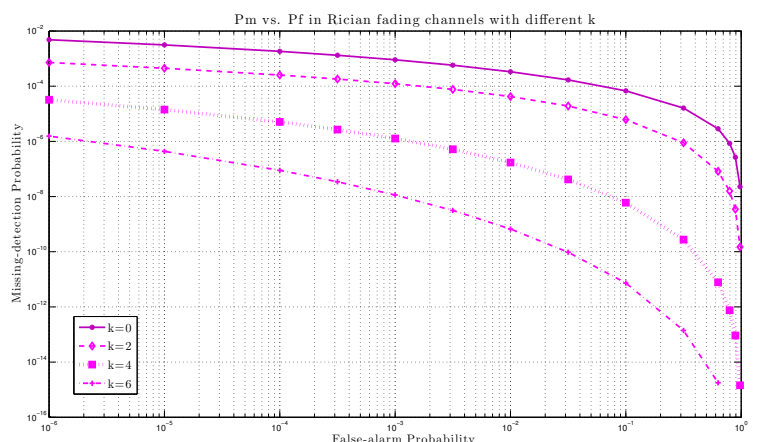

Fig. 6: Complementary ROC curves for Rician fading channel for different values of Rician factor $k(N=5$,

$$
\bar{\gamma}_{R}=8 \mathrm{~dB} \text { and } u=5 \text { ). }
$$

[2] J. Mitola and G. Maguire, "Cognitive radio: Making software radios more personal," IEEE Personal Communications, vol. 6, no. 4, pp. 1318, August, 1999.

[3] I. Akyildiz, W. Lee, M. Vuran, and S. Mohanty, "NeXt generation/dynamic spectrum access/cognitive radio wireless networks: A survey," Computer Networks, vol. 50, no. 13, pp. 2127-2159, September, 2006.

[4] C. Sun, W. Zhang, and K. Letaief, "Cluster-based cooperative spectrum sensing in cognitive radio systems," in Proc. of the IEEE International Conference on Communications (ICC'07), pp. 2511-2515, Glasgow, Scotland, June, 2007.

[5] W. Zhang, R. Mallik, and K. Letaief, "Cooperative spectrum sensing optimization in cognitive radio networks," in Proc. of the IEEE International Conference on Communications (ICC'08), pp. 3411-3415, Beijing, China, May, 2008.

[6] L. Yang, M. Kang, and M.-S. Alouini, "On the capacity-fairness tradeoff in multiuser diversity systems," IEEE Transactions on Vehicular Technology, vol. 56, no. 4, pp. 1901-1907, April, 2007.

[7] M. Yacoub, Foundations of Mobile Radio Engineering. CRC Press, Inc. Boca Raton, FL, USA, 1993.

[8] F. Digham, M.-S. Alouini, and M. Simon, "On the energy detection of unknown signals over fading channels," IEEE Transactions on Communications, vol. 55, no. 1, pp. 21-24, May, 2007.

[9] M. Nakagami, "The m-distribution- A general formula of intensity distribution of rapid fading," in Statistical methods in radio wave propagation (W. G. Hoffman, ed.), pp. 3-36, Pergamon Press, Oxford, England, 1960.

[10] A. Nuttall, "Some Integrals Involving the $Q_{M}$-Function," Naval Underwater Systems Center (NUSC) Technical Report, May 1974.

[11] H. Solomon and M. Stephens, "Distribution of a sum of weighted chi-square variables," Journal of the American Statistical Association, vol. 72, no. 360, pp. 881-885, June, 1977. 